\title{
Analysis of Movement Quality in Full-Body Physical Activities
}

\author{
RADOSLAW NIEWIADOMSKI, KSENIA KOLYKHALOVA, STEFANO PIANA, \\ PAOLO ALBORNO, GUALTIERO VOLPE, and ANTONIO CAMURRI, University of Genoa
}

Full-body human movement is characterized by fine-grain expressive qualities that humans are easily capable of exhibiting and recognizing in others' movement. In sports (e.g., martial arts) and performing arts (e.g., dance), the same sequence of movements can be performed in a wide range of ways characterized by different qualities, often in terms of subtle (spatial and temporal) perturbations of the movement. Even a non-expert observer can distinguish between a top-level and average performance by a dancer or martial artist. The difference is not in the performed movements-the same in both cases-but in the "quality" of their performance.

In this article, we present a computational framework aimed at an automated approximate measure of movement quality in full-body physical activities. Starting from motion capture data, the framework computes low-level (e.g., a limb velocity) and high-level (e.g., synchronization between different limbs) movement features. Then, this vector of features is integrated to compute a value aimed at providing a quantitative assessment of movement quality approximating the evaluation that an external expert observer would give of the same sequence of movements.

Next, a system representing a concrete implementation of the framework is proposed. Karate is adopted as a testbed. We selected two different katas (i.e., detailed choreographies of movements in karate) characterized by different overall attitudes and expressions (aggressiveness, meditation), and we asked seven athletes, having various levels of experience and age, to perform them. Motion capture data were collected from the performances and were analyzed with the system. The results of the automated analysis were compared with the scores given by 14 karate experts who rated the same performances. Results show that the movementquality scores computed by the system and the ratings given by the human observers are highly correlated (Pearson's correlations $r=0.84, p=0.001$ and $r=0.75, p=0.005$ ).

CCS Concepts: • Computing methodologies $\rightarrow$ Activity recognition and understanding; Model development and analysis; • Applied computing $\rightarrow$ Arts and humanities;

Additional Key Words and Phrases: Gesture analysis, movement quality, full-body movement, karate, dance

ACM Reference format:

Radoslaw Niewiadomski, Ksenia Kolykhalova, Stefano Piana, Paolo Alborno, Gualtiero Volpe, and Antonio Camurri. 2019. Analysis of Movement Quality in Full-Body Physical Activities. ACM Trans. Interact. Intell. Syst. 9, 1, Article 1 (February 2019), 20 pages.

https://doi.org/10.1145/3132369

This research has received funding from the European Union's Horizon 2020 research and innovation program under grant agreement no. 688269 (TELMI) and no. 688865 (WhoLoDanceE).

Authors' addresses: R. Niewiadomski, K. Kolykhalova, S. Piana, P. Alborno, G. Volpe, and A. Camurri, InfoMus Lab, DIBRIS, University of Genoa, viale Causa 13, 16-145 Genoa, Italy.

This work is licensed under a Creative Commons Attribution International 4.0 License.

(c) 2019 Copyright held by the owner/author(s).

2160-6455/2019/02-ART1

https://doi.org/10.1145/3132369

ACM Transactions on Interactive Intelligent Systems, Vol. 9, No. 1, Article 1. Publication date: February 2019. 


\section{INTRODUCTION}

Humans have capabilities to develop very fine skills to perform several complex full-body activities. They are also willing to spend a lot of effort and time to train themselves to gain these skills. When they have learned the bases of an activity, soon they are not satisfied by its mere realization. They enrich the functional aspect of a physical activity (e.g., moving, changing location) with qualitative features, aiming at improving communication or at an optimization of the movement effect. These qualitative features are usually expressed in terms of fine-grain perturbations of spatial and temporal movement dynamics. For example, ice skating is not only a way of changing location in winter conditions, but a skating performance can be evaluated with respect to its quality in addition to the increase of effectiveness of the action, e.g., improved velocity, reduction of fatigue, and easier achievement of more complex actions.

In this article, we focus on the computational analysis of movement quality of physical activities. Movement quality is for us a measure of the general excellence of a specific movement realization in terms of the judgment given by an expert observer. In our computational approach, it consists of three major components: Biomechanical Efficiency, Shape, and Intrapersonal Synchronization and includes several aspects such as postural control, coordination, and balance. Movement quality can be considered in the context of different activities, such as dance (e.g., classic ballet and contemporary dance) (Alexiadis et al. 2011), sport (e.g., martial arts, skating, gymnastics), crafting (e.g., pottery), yoga, tai-chi, and music performance (Mion and Poli 2008). Automatic evaluation of movement quality may have several important applications. It can be used, for example, for more efficient training. Using technology, apprentices may discover even small imperfections that can decrease the effectiveness of the performance or even cause injuries (Brodie et al. 2008; Dowling et al. 2011). Technology may also be used in competitions (e.g., dance, gymnastics, or ice skating) for supporting a panel of judges in their assessment (Daz-Pereira et al. 2014). Further, advances in knowledge on movement quality can contribute to research in the understanding of aesthetic experience from a computational perspective, in contributing to artistic languages, and in several other scientific domains. Consequently, there exists a need for developing computer systems able to automatically assess movement quality of physical activities.

In this article, we address this necessity by introducing a computational framework for evaluating the overall movement quality of a performance. Starting from different data sources (e.g., MoCap data) the framework computes several low-level (e.g., single-joint velocity peaks) and highlevel (e.g., synchronization between different limbs) features. It can be instantiated to evaluate movement quality in different activities. Moreover, the computation of movement quality is not restricted to single body movements; the framework computes an overall movement quality score on a sequence of movements or even on a whole performance.

In the second part of the article, we present a system representing a specific instance of our framework aimed at evaluating the movement quality of predefined sequences of movements performed by athletes. Karate is taken as a case study for investigation mainly because karate training is based on katas, that is, choreographies useful for practicing certain sequences of movements and actions, and to improve coordination, concentration, and muscle memory. Katas are particularly suitable because the sequence of actions in a kata is precisely defined. Thus, it is relatively easy to collect several performances of the same sequence of movements by different athletes and create a dataset in which the sequences differentiate mainly by movement quality. We built such a dataset to evaluate our system. Further, katas include complex movements whose movement quality might be influenced by various factors, such as correct coordination, posture, and biomechanical efficiency. In our system, full-body MoCap data are used by several modules developed on purpose, which measure different aspects of the kata performance. In order to assess the 
performances of our system, we compare the computed movement quality against perceived quality ratings provided by experts in the field.

The remainder of this article is structured as follows. The next section describes the state of the art in measuring movement quality. A model of movement quality is then discussed in Section 3. Section 4 describes our computational framework. Section 5 presents and evaluates a system and an instance of the framework, performing the analysis of motion capture data of karate athletes. Section 6 shows how the framework can be applied to other application scenarios, focusing on dance and musical instrument training. We present our conclusions in Section 7.

\section{RELATED WORK}

Several ad-hoc solutions were proposed to evaluate the quality of a performance in complex physical activities. Alexiadis et al. (2011) described a system that automatically evaluates dance performance against a reference gold standard performance. The system compares joint positions and velocities. The method is based on motion acquisition via Kinect and on the application of appropriate quaternionic signal-processing techniques for temporally synchronizing, spatially aligning, and comparing signals characterizing the dance performance. Muneesawang et al. (2015) used Dynamic Time Warping (DTW) to provide a dance student with visual feedback on her performance in real time. In this system, a dancer's gestures are first captured with Kinect, recognized, and isolated. Then, the gestures are compared with a prerecorded template (i.e., the same choreography performed by a dance teacher). Finally, visual feedback is given to the dance student in the form of a colored skeleton, in which the color of a limb represents the IDTW distance (i.e., the amount to which the student's performance matches the dance template). Kitsikidis et al. (2014) proposed a fuzzy logic model to detect the quality of a dancer's performance, considering the evaluation by an expert of traditional Greek dances as baseline. For this purpose, they exploited multiple Kinect depth sensors to compute the position of body joints. Next, a two-layer fuzzy inference system was proposed in which low-level features obtained from raw tracking data and high-level motion recognition probabilities provided by a separate motion analysis module are used in parallel as input for the evaluation of the dancer's performance. Barrett et al. (2014) proposed a technique for estimating gesture accuracy within the context of a motion-based rehabilitation video game. In their scenario, players have to imitate certain reference gestures. The player's performance is estimated by means of the degree of similarity between performed and reference gestures in terms of a vector of Euler angles. Daz-Pereira et al. (2014) proposed an algorithm for assigning quality judgment scores of rhythmic gymnastic movements. Their method assigns a quality judgment score that is related to the distance between the spatio-temporal trajectories of the performed movement with respect to prerecorded template movements collected in a training set. More specifically, the algorithm consists of a training and prediction phase. The training phase uses a set of videos that are segmented in single movements, each labeled with its corresponding quality judgment score. Next, for each movement, the corresponding image sequence is converted to a Motion Vector Flow Instance template sequence, and a Principal Component Analysis (PCA) eigenspace is found by combining all such template sequences included in the training set. In the prediction phase, a new video shot is transformed into a sequence of templates that are projected into the PCA eigenspace defined previously. A quality judgment score is assigned to the new input video by considering how close it is to the annotated training sequences by using a kNN distance metric. Ilg et al. (2003) presented a learning-based method for the estimation of skill levels from sequences of complex movements in sports. They used a hierarchical algorithm for computing spatio-temporal correspondences between sequences of complex body movements. Their approach was applied to karate performances by seven athletes. Using spatio-temporal morphable models, the authors characterized individual movement elements by linear combination of learned example patterns. 
The coefficients of such linear combinations can be exploited as descriptors of the movement style. Bianco and Tisato (2013) proposed an automatic system for analysis of movement quality of single karate actions. They assumed that one of the key factors to evaluate the effectiveness of a movement is the relative position of body joints. Using an angular representation of the skeletal data, they extracted descriptive features and classified key poses using machine learning techniques. Movement quality was assessed by comparing the temporally aligned recognized key poses with a set of exemplar movements.

In the specific framework of martial arts, Chua et al. (2003) developed a virtual reality system for tai-chi training. The system, composed of a lightweight belt-worn video receiver and of a head-mounted display, tracks full-body movements and produces an animated representation in virtual reality. The authors showed that such a system could improve training for a full-body motor task in a virtual training scenario in which a virtual teacher is performing directly in front of real students. Witte et al. (2012) proposed a method to identify similarities and differences in coordination between the movements of karate performers. Vieten and Riehle (2005) studied movement quality of kicks of nine subjects from data recorded by five digital cameras. Cynarski et al. (2014) proposed developing a mathematical model of karate actions aiming at comparing novice and expert athletes. Their preliminary results consist of the kinematic analysis of punches in terms of velocity and acceleration. Timmi (2012) developed a system called Virtual Sensei to evaluate the kinetic energy of an action. The system is based on Kinect and can evaluate the efficiency of single actions by measuring the instantaneous kinetic energy of the limbs and of the full-body.

\section{MODELING PHYSICAL MOVEMENT QUALITY}

Several aspects may influence the evaluation of a physical activity, such as cultural background or personal experience of the observer. Physical activities may have different goals, such as narration (e.g., to communicate the plot of a story by the sequences of figures of a classical ballet), expression (e.g., to convey emotions with a posture in a music performance or to communicate other aesthetic feelings in contemporary dance), and physical efficiency (e.g., to reduce the physical effort to improve sportive performance, e.g., in ski-jumping). These goals are often not disjunctive; for example, the movements of a classic ballerina are simultaneously narrative (they communicate the plot of a ballet) and expressive because they elicit emotional reactions in spectators. When teaching a new exercise to students, the fitness trainer performs it, giving emphasis to the difficult parts (e.g., by slowing down their execution) to communicate properly their details and complexity. When learning to play a music instrument, students learn the instrumental gestures required to play in a physically efficient manner (e.g., pressing keys, stepping on pedals) and how to convey expressiveness, for example, through ancillary gestures (Nusseck and Wanderley 2009). Despite the variety of goals and meanings of physical activities, in our computational approach, we assume that each movement (or a sequence of movements) can be evaluated with respect to its quality, that is, the general excellence of the movement performance in terms of Biomechanical Efficiency, Shape, and Intrapersonal Synchronization.

Biomechanical efficiency means whether movement is efficient according to biomechanical laws (e.g., minimum jerk (Flash and Hogans 1985) or two-thirds power law (Viviani and Schneider 1991)). Biomechanical efficiency helps avoid injuries and waste of energy. In sport, biomechanically efficient movements approach theoretical maximal effectiveness (in the sense of velocity or force) and minimalize energy effort.

Shape concerns postural aspects of the movement performance, for example, whether the appropriate posture is maintained. It focuses on the static shapes (postures) that the body takes as well as how the body changes from one shape to another during movement. 


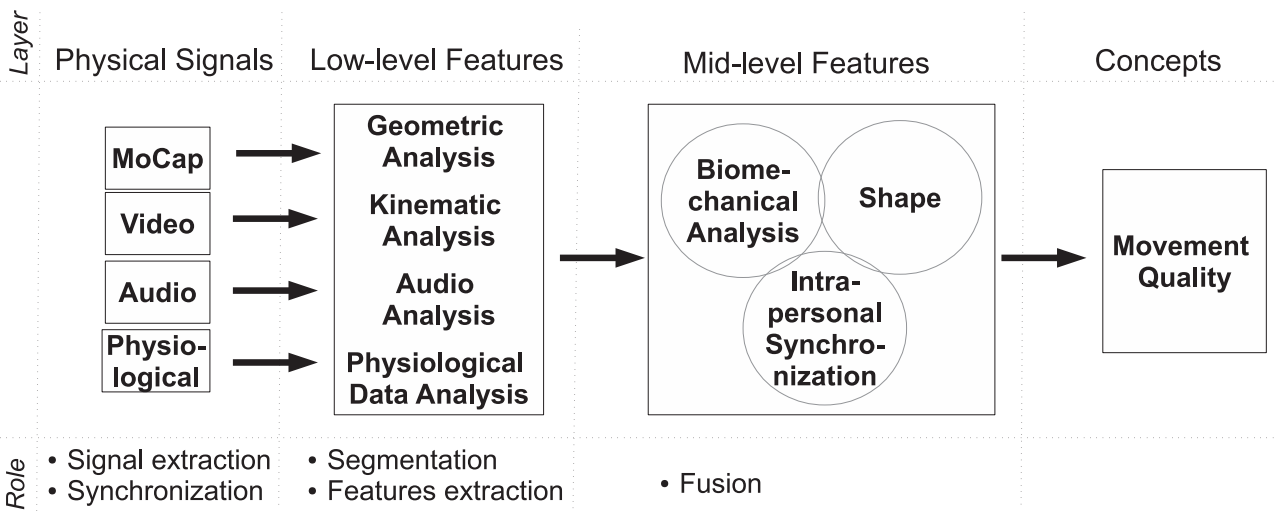

Fig. 1. Our computational framework for the analysis of movement quality in full-body physical activities.

Intrapersonal synchronization focuses on body limb coordination and on the time relationship between the movement of different parts of the body, for example, whether arms move synchronously.

Our definition of movement quality focuses on the technical aspects of a movement performance. We exclude subjective factors that may influence the perception of movement quality at the individual level, for example, cultural background of the observer. Moreover, the correct performance of a movement (i.e., a high-quality movement) is usually related to the goals of the movement (e.g., an excellent performance in terms of intrabody synchronization may evoke positive aesthetic feelings in spectators), but movement quality is not evaluated in goal-oriented terms. Modeling goal-oriented effectiveness of a physical activity (e.g., whether a particular movement conveys an intended affective meaning or not (Pasch et al. 2009)) is out of the scope of this work.

\section{COMPUTATIONAL FRAMEWORK}

In order to measure movement quality, we designed a computational framework consisting of four layers and several modules (see Figure 1). This is grounded on a conceptual framework conceived for analysis of expressive content conveyed by full-body movement and gesture (Camurri et al. 2004, 2016b).

The first layer of our framework-the Physical Signals Layer-consists of modules that capture and preprocess sources of data from different modalities. Below are more details about these modules.

- MoCap Module: It retrieves the 3D positions of body joints from a motion capture system, applies basic processing techniques (e.g., signal filtering), and computes basic kinematic features, such as velocity or acceleration.

- Video Module: It receives video streams from one or more video cameras and/or RGB-D sensors and possibly runs basic video-processing techniques (e.g., background subtraction and motion tracking).

- Audio Module: It captures audio streams from one or more environmental or on-body microphones and possibly runs basic audio-processing techniques (e.g., denoising). In our system, we focus on nonverbal audio.

- Physiological Signals Module: It retrieves data from physiological sensors - such as respiration or skin conductance response sensors - and applies basic processing techniques (e.g., signal filtering). 
The Low-Level Features Layer consists of modules that compute basic features describing movement quality. Such modules are regrouped into different sets performing different analyses:

- Geometric Analysis, for example, computes distances and angles between joints.

- Kinematic Analysis computes movement trajectories as well as basic kinematic information (e.g., acceleration peaks and kinetic energy).

- Audio Analysis performs extraction and analysis of acoustic features, for example, Mel Frequency Cepstral Coefficients (MFCC) (Davis and Mermelstein 1980; Zheng et al. 2001), main frequency F0, or volume.

- Physiological Data Analysis performs physiological signal processing and analysis, for example, signal peak detection and signal periodicity.

At the Mid-Level Features Layer, quality is analyzed with respect to its three major components discussed in the previous section: Biomechanical Analysis, Shape, and Intrapersonal Synchronization.

Finally, on the top, the Concepts Layer is composed of one module that computes overall movement quality. The aim of this level is to merge the different facets of quality into one meaningful value. Since overall movement quality is related to the goals of each specific activity, different fusion models should be used for different activities. For example, Intrapersonal Synchronization may weight more in classic ballet, whereas Biomechanical Efficiency may weight more in sport activities that require a lot of physical effort.

\section{CASE STUDY: A SYSTEM FOR ANALYSIS OF MOVEMENT QUALITY IN KARATE PERFORMANCES}

In this section, we propose a system for the analysis of movement quality in karate performances. The system implements the framework presented in the previous section. Next, we apply the system to analyze the kata performances by seven martial artists and collect the movement quality scores computed by the system for these performances. In the last step, we evaluate the system against the human experts who ranked the same set of kata performances.

\subsection{Material: Kata Performances}

Martial arts (e.g., karate, taekwondo, and so on) use katas, that is, forms or patterns helping to practice certain moves (e.g., kicking combinations) and to improve physical conditioning, muscle memory, focus/concentration, and balance. Katas help to practice "offensive" or "defensive" techniques, for example, striking an imaginary opponent's neck at the correct height and angle. It is important to develop automatic techniques to measure a kata's performance quality, for evaluating the performance of karate adepts and for avoiding developing wrong movement habits. Some aspects of a kata's performance quality are intuitive and easily observable even by non-experts, such as maintaining a correct posture. Maintaining the appropriate posture means to put the body in natural alignment, increase energy, reduce muscle strain, and prevent repetitive strain injuries.

The most popular and teachable style in martial arts schools is Shotokan, which consists of 26 katas. For the purpose of this case study, two Shotokan katas were chosen: Heian Yondan and Bassai Dai. The choice was based on discussions with karate experts who helped in picking the sequences of movements most suitable for investigation. Moreover, the experts indicated essential aspects of each technique. The two katas are different in movements, speed, rhythm, and difficulty. The first kata focuses on leg techniques, the second on hand techniques. Heian Yondan is introduced at the green belt (6th Kyu) skill level as one of the important exercises and consists of 27 karate movements (counts). Bassai Dai is more difficult. It is introduced at the brown belt (3rd Kyu) skill level and often performed for black-belt examination. It consists of 38 karate movements and 
is characterized by many switching arm-blocking combinations. The most difficult part of Bassai Dai is hip motion. Fast, strong, and snappy hip rotations are needed to perform a good Bassai.

\subsection{Methodology and System Design}

Our main objective in this case study is not to perform the most accurate biomechanical analysis of each movement but rather to build a versatile system that can be used to compute the overall movement quality of a performance. For this purpose, we developed a set of measures that estimate different aspects of a good kata performance and that can be implemented by a relatively simple system. When considered all together, these measures, at least in the ideal case, should provide a good approximation of the experts' perception of the movement quality of the same performance. This objective can be obtained by an appropriate choice of features and their computation. We do not aim, however, to create a complete biomechanical model of the optimal realization of a technique. Indeed, we intentionally use the data of athletes with different levels of experience, whose performances are not always of the highest quality. Moreover, we do not claim to estimate the overall skill level of the athlete, as a single performance may not correspond to the athlete's overall level of experience. We also intentionally avoid recognition of single actions or postures. A different approach to analysis of movement quality consists of building ad-hoc systems (see previous works in Section 2) that detect and evaluate single techniques or postures (e.g., by using machine learning techniques). Such systems might be more efficient in the evaluation of the quality of the specific kata, but they would be limited only to the set of techniques on which training was performed.

To design our system, we used the official guidelines (FIJLKAM 2013) adopted by the Italian Judo Wrestling Karate and Martial Arts Federation (FIJLKAM $)^{1}$. This 60-page document defines the rules of the organization and criteria for evaluating karate performances during competitions. In particular, it specifies 6 main criteria for the evaluation of a kata: Technics, Power, Kime, Rhythm, Expressivity, and Synchronization. Technics consists of the optimal performance of each single motor action and includes factors such as appropriate intrabody coordination, dynamic and static equilibrium, stability, and lack of parasite movements. Power is related to the amount of kinetic energy produced when performing a technique. Good Kime means that each technique should finish with a very short contraction of isometric muscles, which results in strong deceleration and lack of parasitic movement (such as wobbliness or vibrations). Rhythm is the measure of the appropriate interlacing of quick with slow sequences of movements. Expressivity corresponds to the transmitted meaning of a technique, which is perceivable by observers, for example, beyond the biomechanical efficiency of the movement. The last criterion-Synchronization-is used to evaluate the interpersonal synchronization in a group of martial artists.

To define the mid-level features of our system, we focused on the first four criteria. In particular, Expressivity does not correspond to our definition as it includes subjective characteristics related to the personality of the performer, and (interpersonal) Synchronization cannot be measured on a single martial artist. Additionally, we split the first category (i.e., Technics) into three subcategories: Stability, Posture, and (Intrapersonal) Coordination. Consequently, we use 6 criteria: Stability (C1), Posture (C2), Power (C3), Kime (C4), Rhythm (C5) and Coordination (C6). Note that these criteria correspond to the 3 macro mid-level classes defined in the previous sections: C5 and C6 correspond to Intrapersonal Synchronization in our model of physical movement quality and corresponding computational framework, $\mathrm{C} 2$ and $\mathrm{C} 4$ correspond to Biomechanical Efficiency, and $\mathrm{C} 1$ and $\mathrm{C} 2$ are related to Shape.

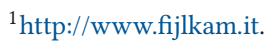




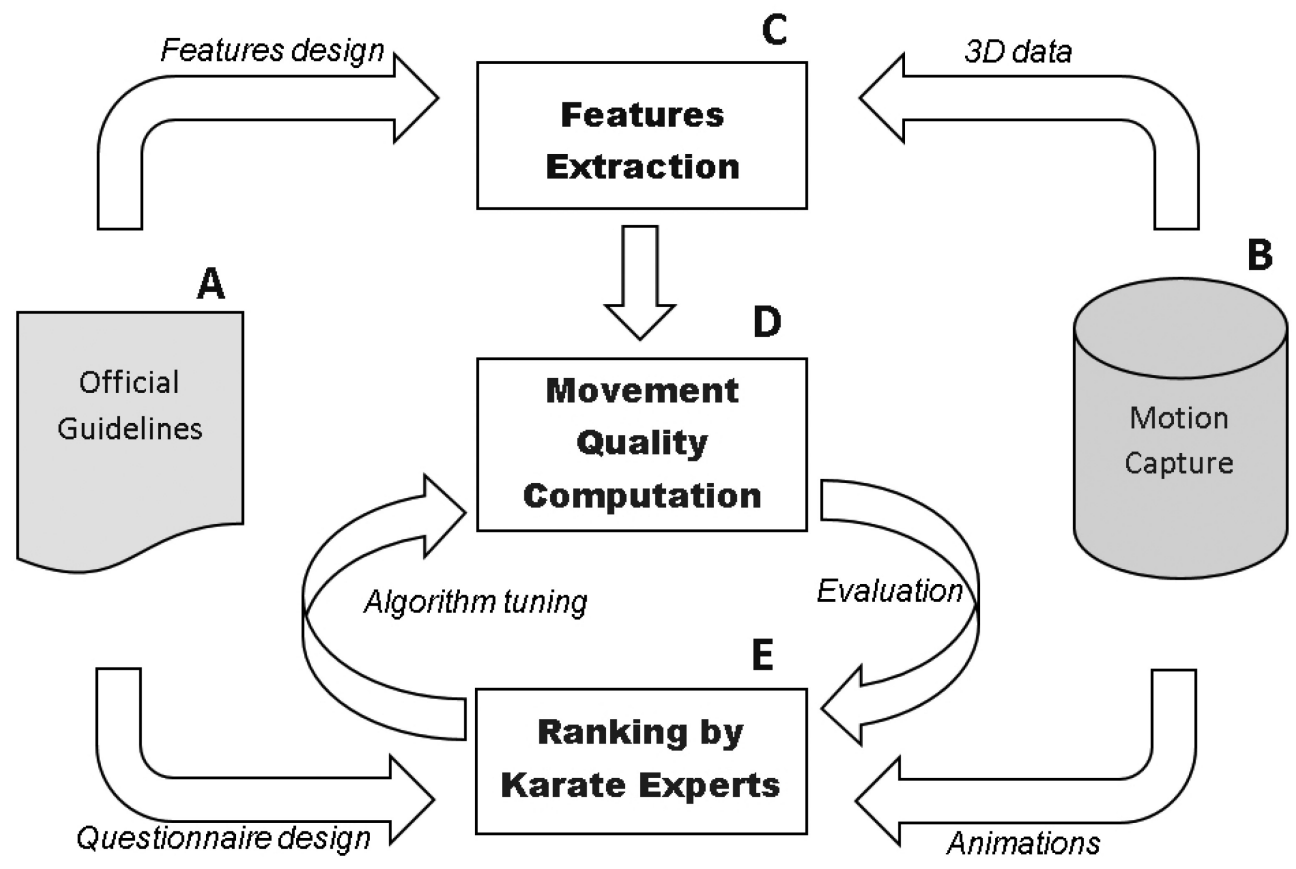

Fig. 2. A summary of the methodology applied in the design and evaluation of the system developed for the specific case study of analysis of movement quality in karate performances.

Figure 2 presents an overview of the methodology that we adopted. Criteria C1 to C6 (Figure 2(A)) are used to define low-level features (Figure 2(C)), which are extracted to compute the overall movement quality (Figure 2(D)). At the same time, the same set of criteria (Figure 2(A)) is used by a group of karate experts (Figure 2(E)), who watched the animations of the motion capture data of the recorded performances (Figure 2(B)) and ranked them. The motion capture dataset consists of the data of two different kata performances by seven karate adepts. These data are used to create the animations displayed to the experts for obtaining the scores (Figure 2(E)) and as input to our system (Figure $2(\mathrm{C})$ ). In the final step, the scores that the experts assigned (Figure 2(E)) are used to tune the system (Figure 2(D)) and to evaluate it.

\subsection{System Architecture}

The system architecture is presented in Figure 3. In this case study, we use just one data source: full-body MoCap. The low-level features layer computes features that characterize different aspects of movement quality, as discussed in the previous section, for example, coordination between the two hands, stability of the barycenter of the performer, straightness of the back, and so on. At the mid-level features layer, the low-level features contribute to the computation of the six mid-level features $C 1$ to $C 6$. At the top level, the Concepts layer is composed of one single module: the output that this module computes is the final movement-quality score.

5.3.1 Physical Signals Layer. We used high-precision MoCap data. This consists of the 3D positions of 25 markers placed on the body. The positions of the markers are described in Table 1.

5.3.2 Low-Level Features Layer. Below, we describe the modules that we developed to compute 16 low-level features $\left(F_{1} \ldots F_{16}\right)$ quantitatively describing movement in the two selected katas. In particular, features $F_{1}, F_{4}$, and $F_{5}$ contribute to the Mid-Level Stability component; features $F_{3}, F_{6}$, 


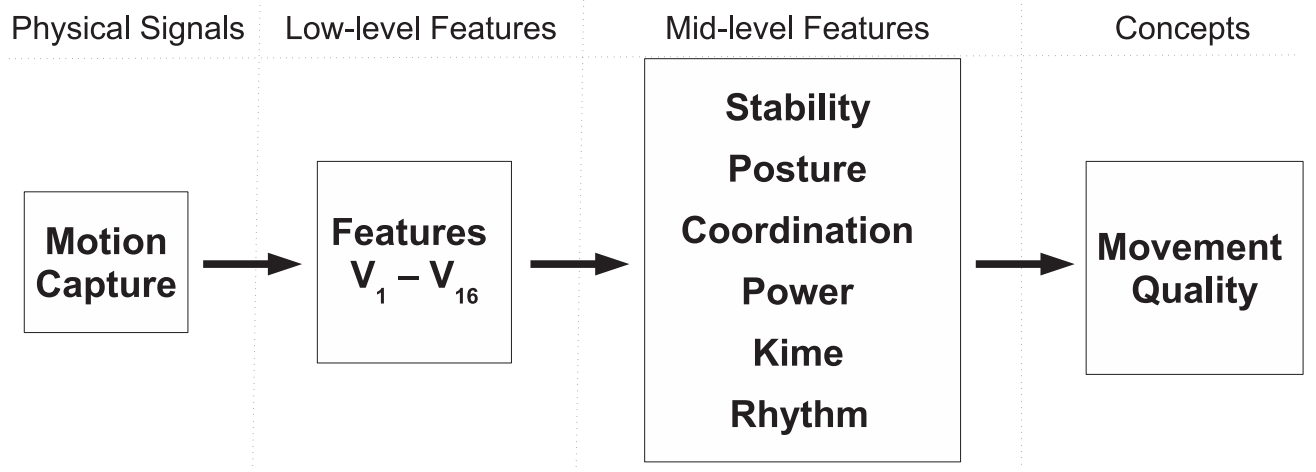

Fig. 3. Instance of the system for evaluating quality of a karate performance.

Table 1. Label List

\begin{tabular}{l|l|l|l|l|l}
\hline$\#$ & Marker Location & Label & $\#$ & Marker Location & Label \\
\hline \hline 1 & Back Head & BKHD & 14 & Right Pinkie Finger & RPNK \\
2 & Right Front Head & RFHD & 15 & Left Pinkie Finger & LPNK \\
3 & Left Front Head & LFHD & 16 & Right Front Hip & RFHP \\
4 & C7 on the Spine & C7 & 17 & Right Back Hip & RBHP \\
5 & Neck & NCK & 18 & Left Front Hip & LFHP \\
6 & Right Shoulder & RSHD & 19 & Left Back hip & LBHP \\
7 & Left Shoulder & LSHD & 20 & Right Knee & RKNE \\
8 & Right Elbow & RELB & 21 & Left Knee & LKNE \\
9 & Left Elbow & LELB & 22 & Right Front Ankle & RFAK \\
10 & Right Wrist & RWRS & 23 & Right Back Ankle & RBAK \\
11 & Left Wrist & LWRS & 24 & Left Front Ankle & LFAK \\
12 & Right Index Finger & RIND & 25 & Left Back Ankle & LBAK \\
13 & Left Index Finger & LIND & & & \\
\hline
\end{tabular}

and $F_{7}$ contribute to the Posture component. Features $F_{8} \ldots F_{10}$ correspond to Coordination, $F_{11}$ to Power, and $F_{12} \ldots F_{15}$ to Kime. Finally, features $F_{2}$ and $F_{16}$ are examples of Rhythm analysis. All of the modules work on data segments of arbitrary duration. Each module outputs one value for the whole input segment.

$F_{1}$ : a general measure of equilibrium. According to the official guidelines, any loss of equilibrium is a severe error that influences the evaluation of the performance. To detect equilibrium loss, we measure the projection of the barycenter of the body on the rectangular area defined by the performer's feet. The algorithm first detects, for each frame, whether both feet are on the ground by checking the values of the vertical component of markers $L F A K=\left(x_{l}, y_{l}, z_{l}\right)$ and $R B A K=\left(x_{r}, y_{r}, z_{r}\right)$. For each frame, it then defines a rectangle $Z$ with four corners: $\left(x_{l}, z_{l}\right),\left(x_{l}, z_{r}\right)$, $\left(x_{r}, z_{r}\right)$, and $\left(x_{r}, z_{l}\right)$, and measures the distance between the barycenter $B=\left(x_{b}, y_{b}, z_{b}\right)$ projected on the $2 \mathrm{D}$ plane defined by the feet positions and the center of the $Z$ rectangle. The smaller the distance, the better the equilibrium. The computed measure of equilibrium is the variance of such a distance over the analyzed segment:

$$
F_{1}=\operatorname{var}\left(\sqrt{\left(\frac{x_{r}+x_{l}}{2}-x_{b}\right)^{2}+\left(\frac{z_{r}+z_{l}}{2}-z_{b}\right)^{2}}\right) .
$$




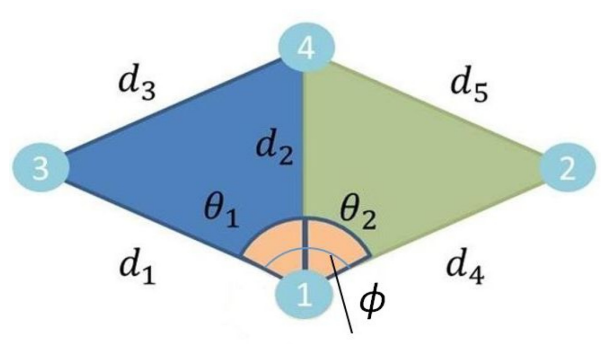

Fig. 4. The angle between the shoulders is computed as the angle $\phi$ between markers RSHD (3), C7 (1), and LSHD (2).

$F_{2}$ : Sharp hip rotations. Hip rotation helps the athlete to push more strength into one's punches. To compute this feature, we measure for each frame $i$ the angular velocity $v^{2} l_{i}$ of the hip plane defined by rigid body $R F H P, L F H P, L B H P, R B H P$, and we take the average angular velocity over the $n$ frames of the analyzed segments:

$$
F_{2}=\frac{\sum_{i}^{n} \text { vel }_{i}}{n}
$$

$F_{3}$ : Low torsion between hips and shoulders. During the execution of a kata, the face and hip should keep the same direction. The degree of body torsion is computed for each frame $i$ as the angle $\phi_{i}$ between the body planes of shoulders (markers: RSHD, LSHD , C7, NCK) and hips (markers: RFHP, $L F H P, L B H P, R B H P$ ). Torsion is the angle $\phi_{i}$ between the traversal projections of these two rigid bodies. The lower the value of $\phi_{i}$, the better the movement quality. Thus, feature $F_{3}$ is computed as the average value of $\phi_{i}$ over the $n$ frames of the analyzed segments:

$$
F_{3}=\frac{\sum_{i}^{n} \phi_{i}}{n}
$$

$F_{4}$ and $F_{5}$ : Stable feet position. The position of the feet should be stable between two subsequent actions. In good performances, there should not be any additional parasitic or supporting feet movements, feet slipping, or vibrations of the lower limbs. For each frame $i$ and for each foot $f \in\{L, R\}$, we compute two values: (1) the kinetic energy of the foot $E_{i}^{f}$ and (2) the kinetic energy of the foot $E f l_{i}^{f}$, filtered with a low-pass filter. Next, we compute $F_{4}$ and $F_{5}$ for each foot separately as

$$
F_{4}=\frac{\sum_{i}^{n} \operatorname{abs}\left(E_{i}^{L}-E f l_{i}^{L}\right)}{\sum_{i}^{n} \max \left(E_{i}^{L}, E f l_{i}^{L}\right)} \quad F_{5}=\frac{\sum_{i}^{n} a b s\left(E_{i}^{R}-E f l_{i}^{R}\right)}{\sum_{i}^{n} \max \left(E_{i}^{R}, E f l_{i}^{R}\right)},
$$

where $n$ is the number of input data frames in the analyzed segment.

$F_{6}$ : Angle between shoulders and neck. According to the guidelines, the karate martial artist should keep the angle between the shoulders (see Figure 4) within a specific range, that is, one has to avoid bending back or moving shoulders forward. Still, the posture is acceptable even if the angle between the shoulders slightly increases owing to performing a punch or a kick. When one hand performs a punch and moves to one direction, the other hand is going to the opposite direction, moving the shoulder to the back, which makes the angle between shoulders bigger. The opposite situation-a decreasing angle-is always considered to be an error. Incorrect postures can be detected by analyzing the variations of the angle between the shoulders and spine. By extracting the 3D positions of markers $L S H D, R S H D, N C K$, and $C 7$, the values of the angles $\theta$ and $\phi$ are calculated according to Equations (5) to (7) (see Figure 4).

$$
d_{3}^{2}=d_{1}^{2}+d_{2}^{2}-2 \cdot d_{1} \cdot d_{2} \cdot \cos \left(\theta_{1}\right) \quad d_{5}^{2}=d_{4}^{2}+d_{2}^{2}-2 \cdot d_{4} \cdot d_{2} \cdot \cos \left(\theta_{2}\right)
$$




$$
\begin{gathered}
\theta_{1}=\operatorname{acos}\left(\frac{d_{1}^{2}+d_{2}^{2}-d_{3}^{2}}{2 \cdot d_{1} \cdot d_{2}}\right) \quad \theta_{2}=\operatorname{acos}\left(\frac{d_{4}^{2}+d_{2}^{2}-d_{5}^{2}}{2 \cdot d_{4} \cdot d_{2}}\right) \\
\phi=\theta_{1}+\theta_{2}
\end{gathered}
$$

For each frame $i$, the corresponding angle $\phi_{i}$ is computed. Then, the average is taken over the $n$ frames of the analyzed segment:

$$
F_{6}=\frac{\sum_{i}^{n} \phi_{i}}{n}
$$

$F_{7}$ : Straightness of the back. The back of the performer has to be straight during the whole activity; therefore, the distance between head and hips has to be almost constant. When the back is straight, the whole body is aligned, producing significantly more power and having a better balance. In order to measure whether the back is straight, the distance Dist $_{i}$ between marker $C 7=\left(x_{1}, y_{1}, z_{1}\right)$ and the midpoint $M=\left(x_{4}, y_{4}, z_{4}\right)$ between markers $\operatorname{LBHP}=\left(x_{2}, y_{2}, z_{2}\right)$ and $R B H P=\left(x_{3}, y_{3}, z_{3}\right)$ is computed for each frame $i$ of an input data segment. The more Dist decreases, the more the posture of the back is incorrect. Since each individual has a different back length, the values of Dist $_{i}$ can be compared only when normalized by considering $\max (D i s t)$, that is, the maximum Dist $_{i}$ an athlete can reach. This maximum value corresponds to the back being straight, for example, at the beginning of the performance. The value of feature $F_{7}$ is then computed as the average value of the normalized distance over the $n$ frames of the analyzed data segment:

$$
F_{7}=\frac{\sum_{i}^{n} \frac{\text { Dist }_{i}}{\max (\text { Dist })}}{n} .
$$

$F_{8}, F_{9}$, and $F_{10}$ : Synchronization between pairs of joints. Intrapersonal synchronization, that is, synchronization of relevant pairs of joints-such as the two wrists or wrist and knee-is important for assessing movement quality. We measure synchronization of pairs of joints using the Event Synchronization (ES) algorithm (Quian Quiroga et al. 2002). This technique compares the timing of occurrence of discrete events in two time series. In our case, events are defined as local maxima of velocity. Essentially, the output of ES is a Synchronization Index (SI), computed on the whole data segment, representing the fraction of event pairs matching in time over the 2 time series. $S I$ ranges in $[0,1]$ : the larger the $S I$, the more synchronized the movements. We compute $S I$ for the following relevant pairs of joints: (1) right wrist/left wrist, (2) right wrist/right knee, and (3) left wrist/left knee:

$$
F_{8}=S I(R W R S, L W R S) \quad F_{9}=S I(R W R S, R K N E) \quad F_{10}=S I(L W R S, L K N E) .
$$

For example, synchronization between the right wrist (RWRS) and left wrist (LWRS) is computed as follows: the coordinates of the RWRS and LWRS markers of each athlete are taken as the input dataset; after computing the time series of velocities for both markers, peaks of velocities-that is, local maxima-are retrieved; two new time series of the same length as the input ones are created, in which local maxima are coded with 1 , while all other values are set to 0 ; ES is finally applied to these time series to compute $F_{8}, F_{9}$, and $F_{10}$.

$F_{11}$ : Kinetic energy of an action. According to the official guidelines for karate juries, the power of single actions is one of the six aspects of a good performance. The higher the velocity of the impact mass is, the more powerful (and consequently more efficient) the action is. To include this aspect in our system, we compute the kinetic energy produced by an action. Given the speed (module of velocity) of all 25 markers, we extract significant movements by applying a threshold to the speed value. The threshold is computed as an average value on a short sliding window. A significant movement starts when speed goes over the applied threshold and ends when speed drops to the same value that it had at the beginning of the movement. Next, instantaneous kinetic 
energy is computed as the mass times the squared speed of the joints. Mass is estimated using anthropometric tables. In the last step, for each significant movement, we search for the maximum of kinetic energy and compute the mean of all maxima energy peaks over the input data segment:

$$
F_{11}=\frac{\sum_{j=1}^{k} \operatorname{maxe}_{k}}{k},
$$

where $k$ is the number of significant movements in the analyzed data segment and maxe $_{k}$ is the maximum of energy in the $j$ th significant movement.

$F_{12}$ and $F_{13}$ : Acceleration/deceleration ratio. A good Kime is another important element of a good performance (see Section 5). Technically, Kime is a short contraction of isometric muscles at the end of an action that results in short and clear deceleration. For instance Emmermacher et al. (2005) and Hofmann et al. (2008) measured the duration of the different phases in a Gyaku-Zuki punch, which is the most used fist punch in karate. According to them, deceleration of the fist can be even half shorter than acceleration. To compute the acceleration/deceleration ratio, we take the instantaneous velocity and acceleration values of markers RWRS and LWRS. Next, we extract significant movements by applying a threshold and consider only the intervals in which absolute velocity is over the applied threshold (see above, the computation of $F_{11}$ ). The threshold is computed as an average value on a short sliding window. For each segment $j$, we compute the duration of the acceleration phase $t_{a c c}^{j}$ and deceleration phase $t_{d e c}^{j}$. The maximum velocity is taken as a separation between the phases. The feature is finally computed separately for each hand $h \in\{L, R\}$ as:

$$
F_{12}=\sum_{j=1}^{k} \frac{1-a b s\left(\frac{t_{a c c}^{j, L}-2 * t_{d e c}^{j, L}}{t_{a c c}^{j, L}+t_{d e c}^{j, L}}\right)}{k} \quad F_{13}=\sum_{j=1}^{k} \frac{1-a b s\left(\frac{t_{a c c}^{j, R}-2 * t_{d e c}^{j, R}}{t_{a c c}^{j, R}+t_{d e c}^{j, R}}\right)}{k}
$$

where $k$ is the number of significant movements in the analyzed data segment.

In the ideal case $F_{12}=1$ and $F_{13}=1$ (i.e., the mean time spent for acceleration is twice as long as the time spent for deceleration).

$F_{14}$ and $F_{15}$ : Sharp punch. Good Kime results also in a clear acceleration and deceleration of the hands without any additional parasitic movement (wobbliness or vibrations) after the punch. We measure the sharpness of the hand movements using an approach similar to the one we used to measure stability of feet position (features $F_{4}$ and $F_{5}$ ). Consequently, for each hand $h \in\{L, R\}$, we compute

$$
F_{14}=\frac{\sum_{i}^{n} a b s\left(E_{i}^{L}-E f l_{i}^{L}\right)}{\sum_{i}^{n} \max \left(E_{i}^{L}, E f l_{i}^{L}\right)} \quad F_{15}=\frac{\sum_{i}^{n} a b s\left(E_{i}^{R}-E f l_{i}^{R}\right)}{\sum_{i}^{n} \max \left(E_{i}^{R}, E f l_{i}^{R}\right)},
$$

where $n$ is the number of frames in the analyzed data segment.

$F_{16}$ : Dynamic symmetry. The last aspect of a good kata performance is the rhythm, which is created by the combination of the different actions and their velocities. According to karate experts, this includes an alternance of very quick movements (showing one's speed and reaction) and slow movement (showing one's good postures and stability). To measure that, we compute dynamic symmetry, a movement feature that we have recently proposed in the context of dance performances (Camurri et al. 2016a). Dynamic symmetry is computed as the minimum between the absolute differences of jerkiness and velocity of the two hands. For each wrist $h \in\{L, R\}$, we separately compute absolute jerk $J^{h}$ and velocity $V^{h}$. Next, we compute the absolute difference of jerk and velocity of the two hands:

$$
D_{J}=a b s\left(J^{L}-J^{R}\right) \quad D_{V}=a b s\left(V^{L}-V^{R}\right) .
$$


Dynamic symmetry is the average minimum between the two above differences over the $n$ frames of the analyzed segments:

$$
F_{16}=\frac{\sum_{i}^{n} \operatorname{Min}\left(1-D_{J i}, 1-D_{V i}\right)}{n} .
$$

5.3.3 Mid-Level and Concepts Layer. At the mid-level, we combine the output values of the features $F_{1} \ldots F_{16}$ in six meaningful mid-level features $C_{1} \ldots C_{6}$ : Stability, Posture, Coordination, Power, Kime, and Rhythm. These six features correspond to the three main components of movement quality according to the definition proposed in Section 3. Each mid-level feature is computed as a weighted sum of the low-level features that explain it, for example, synchronization is a weighted sum of $F_{8} \ldots F_{10}$. The weights were chosen manually by considering the importance of each low-level feature (e.g., we give more importance to the synchronization of the hands rather than to the synchronization of the hand-knee pairs).

To assess the overall movement quality of each athlete, we use the output values of the mid-level features $C_{1} \ldots C_{6}$. The overall movement-quality module computes a global quality score $S_{j}$ of the performance $j$ according to the following equation:

$$
S_{j}=\sum_{k=1}^{6} C_{k} * w_{k},
$$

where $w_{k}$ are the weights that express the contribution of each mid-level feature to the global quality score. At the Concept Layer, weights are computed automatically (see Section 5.5.5 for more details about how the system was tuned).

\subsection{Implementation}

The system was implemented in the EyesWeb XMI ${ }^{2}$ platform. EyesWeb XMI allows for real-time recording, synchronization, and processing of multimodal data. It includes a collection of software modules that are assembled in a visual language to build applications. EyesWeb modules support analysis of nonverbal motor behavior, including motion trackers, real-time extraction, and analysis of motion qualities, trajectory analysis, time-series analysis, machine learning, and analysis of social interaction.

\subsection{Tuning and Evaluation}

For evaluating our system, we collected 3D MoCap data of the two selected katas performed by seven martial artists. Athletes had different level of expertise from amateur (students) to professional level (participant in world championship). Data were processed by our system and one global measure-a movement quality score-for each athlete and performance was obtained. Finally, the output of the system was compared against human observers' ratings of the same set of performances. It is important to note that, in this evaluation, our aim is not to estimate the overall level of experience of the martial artist. First, we believe that it might be impossible to estimate the overall experience from one or two short performances. Second, a single performance may, but does not have to, correspond to the overall experience of an athlete; for example, a highly experienced athlete may realize a bad performance owing to some temporary light indisposition, while an amateur may perform once unexpectedly well. However, we believe that having athletes with different levels of experience, it is easier to collect performances characterized by a different movement quality. Thus, in our system, we aim to evaluate the movement quality of the actual performance and we compare it to the one that the observers perceived.

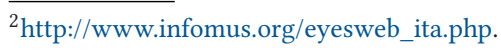


5.5.1 Athletes. A total of 7 athletes $\left(P_{1}-P_{7}\right)$ took part in the study. All athletes received a martial arts education in karate; however, they have different levels of expertise: 2 athletes $\left(P_{1}, P_{5}\right)$ are considered to be highly experienced with more than 15 years of experience. One is also a karate trainer and the other participated in several championships. Two other athletes $\left(P_{2}, P_{3}\right)$ have around 10 years of experience and the remaining 3 athletes have around 5 years of practicing.

5.5.2 Experimental Setup and Method. Data were recorded at the authors' laboratory and included (i) 3D positions of body joints (see Table 1), (ii) the audio signal captured by a microphone located near the performer's mouth and wireless connected to the recording system, and (iii) the video signal of a professional video camera having a frontal view of the capturing area. The Qualisys optical Motion Capture System was used, which consists of 9 high-resolution video cameras. Recordings were carried out by using the Qualisys system's native software Qualisys Track Manager (QTM) v. 2.9. The positions of 25 reflective markers in the 3D space were tracked at a frame rate of $250 \mathrm{~Hz}$. The markers were attached to a black elastic suit on the same joints for each athlete. Motion capture and audio recordings were synchronized using the SMPTE timestamps generated by the professional video camera. The following parameters were set for the video recordings: resolution $1024 \times 576,50 \mathrm{fps}$. CD-quality was used for audio recordings. Each athlete was asked to perform the 2 selected katas twice (Bassai Dai and Heian Yondan). Therefore, a total number of $n=28$ trials was obtained. Athletes could perform katas with their own speed and rhythm. The only requirement was to not exceed the specified area of the floor that was captured by the video camera. The duration of the recordings for kata Bassai Dai is from 71s to 115s. For Heian Yondan, it is from 50s to 97s. More details about the recoding procedure can be found in Kolykhalova et al. (2015).

5.5.3 Rating by Human Observers. Fourteen karate experts (3 females; 64\% Italian, 21\% Polish, average age: 38.8 years, average experience in karate: 16.6 years) ranked the different performances of the Bassai Dai and Heian Yondan kata from the dataset. In the remainder of the article, we use the term observer to refer to the karate experts who participated in this ranking. Each observer was asked to watch 14 animations (i.e., a half of the whole dataset), which were generated from the motion capture data. Each animation consists of one performance of each kata by one of the seven martial artists. Thus, each observer ranked only one performance of a given kata by an artist. The selected animation is a stick-figure visualization of positional data of the 25 markers (see Table 1). To facilitate the task, each animation is composed of two synchronized views of the same performance (i.e., two different virtual cameras were placed on the two opposite sides of the area; see Figure 5). The observers could watch each animation as many times as they wanted, but they had to answer all questions before passing to the next animation and they could not correct their previous answers. The animations do not contain sound and they were displayed in random order. We intentionally used the motion capture data instead of using the videos because we wanted to avoid the observers (1) recognizing their colleagues in the videos and (2) being influenced by the personal characteristics of the athlete (e.g., age). Importantly, the observers used the same information for ranking movement quality as the algorithms do to compute the features. We avoid that humans have some additional information to perform their judgment (e.g., facial expressions) that is not available to the system.

Observers answered the following questionnaire using 5-point Likert scales:

(Q1) What is the overall quality of the performance? (from 1 - very bad to 5 - very good)

(Q2) How good is the performance in terms of stability of the body (e.g., lack of parasitic movement, wobbliness, disequilibrium)? (from 1 -very bad to 5 -very good)

(Q3) How good is the performance in terms of posture (e.g., alignment of the body)? (from 1 - very bad to 5 - very good) 


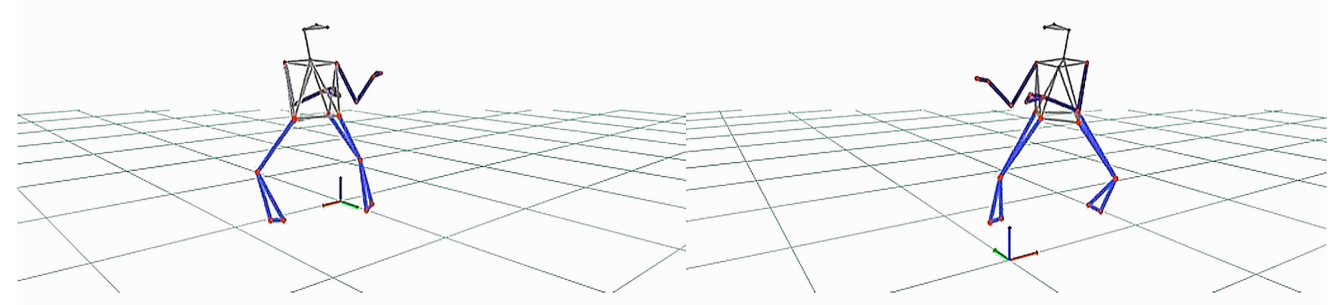

Please, answer all the questions:

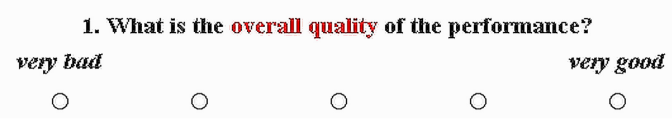

Fig. 5. Interface for animations rankings.

Table 2. Human Observers Rating of Bassai Dai

\begin{tabular}{lccccccc}
\hline Person & Q1 & Q2 & Q3 & Q4 & Q5 & Q6 & Q7 \\
\hline \hline$P_{1}$ & $3.80(1.01)$ & $3.93(1.10)$ & $3.80(1.15)$ & $3.93(1.10)$ & $3.93(1.10)$ & $4.00(1.00)$ & $3.60(1.12)$ \\
$P_{2}$ & $2.92(0.79)$ & $3.17(0.94)$ & $2.92(1.00)$ & $2.58(0.90)$ & $2.67(0.78)$ & $3.33(0.65)$ & $3.17(0.83)$ \\
$P_{3}$ & $2.77(1.09)$ & $2.92(0.86)$ & $3.08(0.76)$ & $2.46(1.13)$ & $2.46(0.97)$ & $3.08(1.04)$ & $3.15(0.55)$ \\
$P_{4}$ & $2.45(1.04)$ & $2.09(0.94)$ & $2.82(1.08)$ & $2.73(0.79)$ & $2.64(0.92)$ & $3.00(1.10)$ & $2.64(1.12)$ \\
$P_{5}$ & $3.67(0.62)$ & $3.67(0.82)$ & $3.67(0.82)$ & $3.73(0.70)$ & $3.53(0.74)$ & $3.73(0.70)$ & $3.80(0.77)$ \\
$P_{6}$ & $4.00(0.77)$ & $3.73(0.90)$ & $3.55(1.13)$ & $4.00(0.77)$ & $3.91(0.94)$ & $3.91(0.70)$ & $3.64(1.03)$ \\
$P_{7}$ & $3.07(1.07)$ & $2.71(1.20)$ & $3.00(0.68)$ & $3.00(1.24)$ & $2.93(0.83)$ & $3.07(1.27)$ & $2.86(1.03)$ \\
\hline
\end{tabular}

(Q4) How good is the performance in terms of kick/punch power? (from 1 - very bad to 5 very good)

(Q5) How good is the performance in terms of Kime? (from 1 - very bad to 5 - very good)

(Q6) How good is the performance in terms of rhythm within the kata (i.e., alternation/transition between quick and slow parts)? (from 1 - very bad to 5 - very good)

(Q7) How good is the performance in terms of intrapersonal coordination (i.e., synchronization of different parts of one's body)? (from 1 - very bad to 5 - very good)

Additionally, all observers provided their level of experience in karate, that is, the color of the belt or dan level. Tables 2 and 3 summarize the average and standard deviation of the scores that the observers provided.

When comparing the results in Tables 2 and 3 with the level of experience of the athletes (see Section 5.5.1), it can be seen that P1 and P5 (highly experienced athletes) received the highest scores for most of the questions. P1 received the highest score 9 out of 12 times (Q2, Q3, Q5, and Q6 in Bassai Dai [BD] and Q2, Q3, Q4, Q5, and Q7 in Heian Yondan [HY]) and P5 received the highest score 2 times (Q7 in BD and Q6 in HY) and the second-highest score 6 times (Q3 in BD and Q2, Q3, Q4, Q5, and Q7 in HY). At the same time, P4 (low experience) received the lowest score 8 times (Q2, Q3, Q6, and Q7 in BD and YH). The performance of P6 (low experience) was scored surprisingly high. $\mathrm{P} 6$ received the best score once (Q4 in $\mathrm{BD})$ and the second-highest score four times (Q2, Q5, Q6, and Q7 in BD). Note that P6's Bassai Dai performances were judged higher than Heian Yondan performances. 
Table 3. Human Observers Rating of Heian Yondan

\begin{tabular}{lccccccc}
\hline Person & Q1 & Q2 & Q3 & Q4 & Q5 & Q6 & Q7 \\
\hline \hline$P_{1}$ & $4.27(0.79)$ & $4.18(0.75)$ & $4.18(1.08)$ & $4.36(0.81)$ & $4.36(0.81)$ & $3.91(1.04)$ & $4.00(0.77)$ \\
$P_{2}$ & $2.57(0.76)$ & $3.00(1.18)$ & $3.00(0.88)$ & $1.93(0.73)$ & $2.29(0.91)$ & $2.93(1.00)$ & $2.86(0.77)$ \\
$P_{3}$ & $2.79(0.97)$ & $3.21(0.97)$ & $3.07(1.07)$ & $2.21(0.97)$ & $2.43(0.94)$ & $2.79(0.97)$ & $3.00(1.11)$ \\
$P_{4}$ & $2.50(1.10)$ & $2.25(1.06)$ & $2.56(0.89)$ & $2.38(0.81)$ & $2.31(0.95)$ & $2.56(0.96)$ & $2.50(0.89)$ \\
$P_{5}$ & $3.58(1.00)$ & $3.50(1.17)$ & $3.67(1.37)$ & $3.67(1.07)$ & $3.58(1.08)$ & $3.92(1.00)$ & $3.58(1.16)$ \\
$P_{6}$ & $3.50(0.63)$ & $3.19(0.91)$ & $3.25(0.86)$ & $3.25(1.13)$ & $3.06(0.77)$ & $3.31(0.79)$ & $3.25(0.86)$ \\
$P_{7}$ & $3.23(0.73)$ & $3.15(0.90)$ & $3.08(0.86)$ & $3.23(1.09)$ & $3.08(0.95)$ & $3.15(1.14)$ & $3.31(0.75)$ \\
\hline
\end{tabular}

If (1) we order the performances according to mean value of observers' scores for each question separately, (2) we associate seven points to the best performance and one point to the lowestranked performance, and (3) we sum the points, then the athlete P1 obtains the highest total score and the second is P5 (both highly experienced). The consecutive athletes are P6 and P7 (low experience), P3, P2 (intermediate level of experience) and P4 (low experience).

This comparison confirms that the technical quality of a performance is only partially related to the experience of the athlete. Inferring quality from the level of experience might be misleading.

5.5.4 Automatic Computation of Mid-Level Features. For each athlete, features $F_{1}$ to $F_{16}$ were computed on the whole kata. Note that even if most modules take as input the data of the whole kata, they actually work more locally by focusing on significant parts of the performance. For example, the energy-related features (see Equation (11)) are computed for those parts of the performance in which the velocity of the joints is above a certain threshold. Thus, slow movements (e.g., those making the transition between two different techniques) are excluded from energy computation.

5.5.5 System Tuning and Validation. The questionnaire intentionally contains redundant data. It can be divided into two parts. The first question, Q1, evaluates overall movement quality. Questions Q2 to Q7 focus on single components of the quality measure, each contributing to the global perceived quality (Q1), and all of them-if considered all together-giving an estimation of Q1. We used this redundancy to tune and evaluate the system. More specifically, we used the scores for Q2 to Q7 to find the weights $w_{k}$ of Equation (16) and the scores for Q1 to evaluate the automatic system against the observers' rankings.

Our approach is structured as follows:

(1) We find a weighted combination of features computed in Section 5.5.4 in order to obtain one (global) movement quality score $S_{j}$ of the performance. The weights $w_{k}$ are computed from the answers to Q2 to Q7.

(2) We compare the global movement quality score $S_{j}$ with the final score (Q1) given by observers (for this purpose, we use the correlation).

Tuning. Here, we describe how we compute the weights $w_{k}, k=1 . .6$ to combine the mid-level features $C_{1}$ to $C_{6}$ into one global quality score. Different mid-level components may contribute differently for each kata. One of the katas focused on hand techniques (Bassai Dai), whereas the other one focused more on leg techniques (Heian Yondan). Thus, we computed the weights $w_{k}$ separately for each kata.

To estimate the contribution of each mid-level quality, we considered the level of the experience of observers (i.e., karate experts). To give more importance to the ranks by more experienced observers, we have performed oversampling: each rank was duplicated $n$ times, where $n$ is the 
Table 4. Weights for Mid-Level Features

\begin{tabular}{lrrrrrrr}
\hline & $\mathrm{C}_{1}$ & $\mathrm{C}_{2}$ & $\mathrm{C}_{3}$ & $\mathrm{C}_{4}$ & $\mathrm{C}_{5}$ & $\mathrm{C}_{6}$ & Constant \\
\hline \hline Bassai Dai & 0.306 & 0.134 & 0.202 & 0.151 & 0 & 0.136 & 0.355 \\
Heian Yondan & 0.0173 & 0.089 & 0.368 & 0 & 0.194 & 0.082 & 0.259 \\
\hline
\end{tabular}

The value 0 means that corresponding predictor was not significant.

level of experience of observer. Next, we apply Linear Regression on scores for Q1 to Q7 using Q1 as the dependent variable on oversampled data. In the case of Bassai Dai, all variables but for one (C5, Rhythm) were significant (see Table 4 for details). The overall model fit was $R^{2}=0.640$. Also, in the case of Heian Yondan, all variables but for one (C4, Kime) were significant (see Table 4 for details). The overall model fit was $R^{2}=0.746$.

Validation. To validate our system, we computed the Pearson's correlation coefficient between a movement quality score $S_{j}$ computed by the algorithm and the overall score (Q1) provided by human observers to the performance $j$.

We checked the correlation for each kata separately. Correlation was high and significant for kata Bassai Dai $(r=0.75, p=0.005)$ and Heian Yondan $(r=0.84, p=0.001)$.

\section{FUTURE WORK: QUALITY OF MUSIC AND DANCE PERFORMANCES}

In addition to martial arts, the computational framework presented here can be applied to other areas in which complex physical activities are performed and a quantitative analysis of movement quality is needed. Two major examples are music and dance, which we will address in our future work.

Dance encompasses complex full-body movements conveying both meaning and expressive content. In dance, analysis of movement quality is obviously of paramount importance. Several low-level and mid-level feature computation modules in our framework can be directly reused depending on the dance genre. For example, synchronization between leg and arm movements is an important aspect in several dance passages in classical and traditional dance; symmetries are relevant in classical ballet. Straightness of the back plays an important role in classical ballet, where it should be maintained during the whole performance.

Playing a music instrument is a complex motor activity requiring years of training. It needs accurate movements of the limbs and the body, fine-grained control of posture, and a high biomechanical efficiency. Moreover, a wrong training approach may cause mechanical problems that develop into injuries (Shan 2003) and levels of excess tension that restrict freedom of movement. It is also well known that movement and gesture play a fundamental role as major conveyors of expressive content in music performance. A quantitative analysis of the quality of motor performance is of great importance in music playing, especially in learning how to play a musical instrument, as a way to assess progress and to avoid injuries.

Analysis of movement quality in dance and music training is currently addressed in two ongoing research projects funded by the European Union under the Horizon 2020 program, the EU-H2020ICT project WhoLoDancE ${ }^{3}$ and the EU-H2020-ICT project TELMI ${ }^{4}$, respectively. The former aims at developing new tools for interactive dance training. The latter aims at designing novel multimodal interaction paradigms and technologies for learning to play a musical instrument, with specific focus on the violin.

Finally, it should be noted that the focus of this article was mainly on the technical quality of movement, rather than on its expressive quality. A more comprehensive evaluation of dance

\footnotetext{
${ }^{3}$ http://www.wholodance.eu/.

${ }^{4}$ http://telmi.upf.edu/.
} 
and music performances will require additional modules to detect the expressive qualities of a movement, for example, whether the movement is fluid, quick, sustained, smooth, rough, and so on. Several algorithms exist for that (e.g., Piana et al. (2016) and Niewiadomski et al. (2015)) and they can be easily included in our framework.

\section{CONCLUSION}

In this article, we presented a computational framework that (i) allows for modeling the concept of movement quality of a physical activity, (ii) allows for applying it to different physical activities in sport, dance, and music playing, (iii) is independent on the data sources, (iv) allows for relatively fast prototyping of applications (as several modules can be reused), and (v) is embedded in the freely available EyesWeb XMI platform.

The computational framework was applied to a concrete case study: we built a systemconceived as an instance of the framework-to evaluate the movement quality of a kata performance in karate. For this purpose, we integrated a set of modules that focus on different aspects of the martial artist's performance, such as biomechanical efficiency and intrapersonal synchronization of movements. The system is based on the official guidelines of the Italian Judo Wrestling Karate and Martial Arts Federation (FIJLKAM). For evaluating the system, a dataset of kata performances was recorded by asking seven athletes with different skill levels to perform them. Each performed two katas. The overall movement quality was automatically computed by our system and compared with the ratings provided by human experts. Results show that the movement quality computed by our system and the ratings that the human observers provided for the same performances are highly correlated ( $r=0.84$ and $r=0.75)$. Note that the system is based on a set of algorithms that do not require training and can work nearly in real time, giving a good estimation of overall movement quality. Obviously, such a system will not make human expert teachers superfluous, but it can be a support tool for karate students during training and performance.

We also discussed how our computational framework can be used to measure and evaluate movement quality in other physical activities, such as dance or music performances. The framework is highly modular, allowing for the reuse of any of the low-level features with the data of other physical activities. Future work will consist of integrating novel sets of modules in the framework. In particular, we will add modules that focus on expressive qualities of movement, for example, to measure whether a movement sequence is fluid or not (Piana et al. 2016). A further extension of the work presented in this article consists of including additional modalities. For example, in the case of karate performances, audio data are a significant source that brings additional information about the quality of the performance, for example, in terms of the performer's nonverbal utterances. The degree of multimodal intrapersonal synchronization (e.g., between body movements and respiration phases; see, e.g., Lussu et al. (2016)) may influence efficiency and thus movement quality. We expect that the integration of new modules that analyze and fuse data from additional modalities will represent a useful extension for our framework.

\section{ACKNOWLEDGMENTS}

Federico Campofiorito, Sensei Marco Soggiu, and his students are kindly acknowledged for providing their performances. We also thank the staff of the Casa Paganini-InfoMus research center of University of Genoa, and professors Marcello Sanguineti and Enrico Puppo for the useful discussions.

\section{REFERENCES}

Dimitrios S. Alexiadis, Philip Kelly, Petros Daras, Noel E. OConnor, Tamy Boubekeur, and Maher Moussa. 2011. Evaluating a dancer's performance using Kinect-based skeleton tracking. In Proceedings of the 19th ACM International Conference on Multimedia (MM'11). ACM, New York, NY, 659-662. 
Christian Barrett, Jacob Brown, Jay Hartford, Michael Hoerter, Andrew Kennedy, Ray Hassan, and David Whittinghill. 2014. Estimating gesture accuracy in motion-based health games. Journal of Virtual Reality and Broadcasting 11(2014), 8 (2014). https://doi.org/10.20385/1860-2037/11.2014.8

Simone Bianco and Francesco Tisato. 2013. Karate moves recognition from skeletal motion. In Proceedings SPIE 8650, ThreeDimensional Image Processing (3DIP) and Applications 2013, Vol. 8650. 86500K-10. http://dx.doi.org/10.1117/12.2006229

Matthew Brodie, Alan Walmsley, and Wyatt Page. 2008. Fusion motion capture: A prototype system using inertial measurement units and GPS for the biomechanical analysis of ski racing. Sports Technology 1, 1 (2008), 17-28. DOI: https://doi.org/10.1002/jst.6

Antonio Camurri, Corrado Canepa, Nicola Ferrari, Maurizio Mancini, Radoslaw Niewiadomski, Stefano Piana, Gualtiero Volpe, Jean-Marc Matos, Pablo Palacio, and Muriel Romero. 2016a. A system to support the learning of movement qualities in dance: A case study on dynamic symmetry. In Proceedings of the 2016 ACM International foint Conference on Pervasive and Ubiquitous Computing: Adjunct (UbiComp'16). ACM, New York, NY, 973-976. DOI : https://doi.org/10. 1145/2968219.2968261

Antonio Camurri, Barbara Mazzarino, Matteo Ricchetti, Renee Timmers, and Gualtiero Volpe. 2004. Multimodal analysis of expressive gesture in music and dance performances. In Gesture-Based Communication in Human-Computer Interaction, Antonio Camurri and Gualtiero Volpe (Eds.). Lecture Notes in Computer Science, Vol. 2915. Springer, Berlin, 20-39.

Antonio Camurri, Gualtiero Volpe, Stefano Piana, Maurizio Mancini, Radoslaw Niewiadomski, Nicola Ferrari, and Corrado Canepa. 2016b. The dancer in the eye: Towards a multi-layered computational framework of qualities in movement. In Proceedings of the 3rd International Symposium on Movement and Computing (MOCO'16). ACM, New York, NY, Article 6, 7 pages. DOI : https://doi.org/10.1145/2948910.2948927

Philo Tan Chua, Rebecca Crivella, Bo Daly, Ning Hu, Russ Schaaf, David Ventura, Todd Camill, Jessica Hodgins, and Randy Pausch. 2003. Training for physical tasks in virtual environments: Tai Chi. In Proceedings of the IEEE Virtual Reality 2003 (VR'03). IEEE Computer Society, Washington, DC, 87-97.

Wojciech J. Cynarski, Wieslaw Wajs, and Antonio Vences-Brito. 2014. Improving the movements of basic karate techniques with the use of motion capture and mathematical modeling. The concept of a research project. Ido Movement for Culture. Journal of Martial Arts Anthropology 14, 1 (2014), 39-53.

S. Davis and P. Mermelstein. 1980. Comparison of parametric representations for monosyllabic word recognition in continuously spoken sentences. IEEE Transactions on Acoustics, Speech, and Signal Processing 28, 4 (Aug 1980), 357-366. DOI : https://doi.org/10.1109/TASSP.1980.1163420

Ariel V. Dowling, Julien Favre, and Thomas P. Andriacchi. 2011. A wearable system to assess risk for anterior cruciate ligament injury during jump landing: Measurements of temporal events, jump height, and sagittal plane kinematics. fournal of Biomechanical Engineering 133, 7 (2011), 071008-071008-7.

M. Pino Daz-Pereira, Ivn Gmez-Conde, Merly Escalona, and David N. Olivieri. 2014. Automatic recognition and scoring of Olympic rhythmic gymnastic movements. Human Movement Science 34 (2014), 63-80. DOI : https://doi.org/10.1016/j. humov.2014.01.001

P. Emmermacher, K. Witte, and M. Hofmann. 2005. Acceleration course of fist push of Gyaku-Zuki. In Proceedings of XXIII International Symposium on Biomechanics in Sports. The People Sport Press.

FIJLKAM. 2013. FIJLKAM, regolamento d'arbitraggio karate. Retrieved January 17, 2019 from http://www.fijlkam.it/karate/ karate-st/regolamenti.html?download=655:2013-regolamento-d-arbitraggio-karate.

Tamar Flash and Neville Hogans. 1985. The coordination of arm movements: An experimentally confirmed mathematical model. Journal of Neuroscience 5 (1985), 1688-1703.

Martin Hofmann, Kerstin Witte, and Peter Emmermacher. 2008. Biomechanical analysis of fist punch Gyaku-Zuki in karate. In 26th International Conference on Biomechanics in Sports. The People Sport Press.

Winfried Ilg, Johannes Mezger, and Martin Giese. 2003. Estimation of skill levels in sports based on hierarchical spatio-temporal correspondences. In Proceedings of Pattern Recognition: 25th DAGM Symposium, Magdeburg, Germany, September 10-12, 2003, Bernd Michaelis and Gerald Krell (Eds.). Springer, Berlin, 523-531. DOI : https://doi.org/10.1007/ 978-3-540-45243-0_67

Alexandros Kitsikidis, Kosmas Dimitropoulos, Erdal Yilmaz, Stella Douka, and Nikos Grammalidis. 2014. Multi-sensor technology and fuzzy logic for dancer's motion analysis and performance evaluation within a 3D virtual environment. In Universal Access in Human-Computer Interaction. Design and Development Methods for Universal Access: 8th International Conference (UAHCI'14). Springer, Cham, 379-390. DOI: https://doi.org/10.1007/978-3-319-07437-5_36

Ksenia Kolykhalova, Antonio Camurri, Gualtiero Volpe, Marcello Sanguineti, Enrico Puppo, and Radoslaw Niewiadomski. 2015. A multimodal dataset for the analysis of movement qualities in karate martial art. In 7th International Conference on Intelligent Technologies for Interactive Entertainment (INTETAIN'15). IEEE, 74-78. DOI : 10.4108/icst.intetain.2015.260039

Vincenzo Lussu, Radoslaw Niewiadomski, Gualtiero Volpe, and Antonio Camurri. 2016. Using the audio respiration signal for multimodal discrimination of expressive movement qualities. In Proceedings of Human Behavior Understanding: 7th International Workshop (HBU'16), Amsterdam, the Netherlands, October 16, 2016, Mohamed Chetouani, 
Jeffrey Cohn, and Albert Ali Salah (Eds.). Springer International Publishing, Cham, 102-115. DOI : https://doi.org/10. 1007/978-3-319-46843-3 7

L. Mion and G. De Poli. 2008. Score-independent audio features for description of music expression. IEEE Transactions on Audio, Speech, and Language Processing 16, 2 (Feb 2008), 458-466. DOI : https://doi.org/10.1109/TASL.2007.913743

Paisarn Muneesawang, Naimul Mefraz Khan, Matthew Kyan, R. Bruce Elder, Nan Dong, Guoyu Sun, Haiyan Li, Ling Zhong, and Ling Guan. 2015. A machine intelligence approach to virtual ballet training. IEEE MultiMedia 22, 4 (Oct 2015), 80-92. DOI : https://doi.org/10.1109/MMUL.2015.73

Radoslaw Niewiadomski, Maurizio Mancini, Gualtiero Volpe, and Antonio Camurri. 2015. Automated detection of impulsive movements in HCI. In Proceedings of the 11th Biannual Conference on Italian SIGCHI Chapter (CHItaly'15). ACM, New York, NY, 166-169. DOI : https://doi.org/10.1145/2808435.2808466

Manfred Nusseck and Marcelo M. Wanderley. 2009. Music and motion: How music-related ancillary body movements contribute to the experience of music. Music perception: An Interdisciplinary fournal 26, 4 (2009), 335-353.

Marco Pasch, Nadia Bianchi-Berthouze, Betsy van Dijk, and Anton Nijholt. 2009. Movement-based sports video games: Investigating motivation and gaming experience. Entertainment Computing 1, 2 (2009), 49-61. DOI: https://doi.org/10. 1016/j.entcom.2009.09.004 Intelligent Technologies for Interactive Entertainment.

Stefano Piana, Paolo Alborno, Radoslaw Niewiadomski, Maurizio Mancini, Gualtiero Volpe, and Antonio Camurri. 2016. Movement fluidity analysis based on performance and perception. In Proceedings of the 2016 CHI Conference Extended Abstracts on Human Factors in Computing Systems (CHI EA'16). ACM, New York, NY, 1629-1636. DOI : https://doi.org/ $10.1145 / 2851581.2892478$

Rodrigo Quian Quiroga, Thoma Kreuz, and Peter Grassberger. 2002. Event synchronization: A simple and fast method to measure synchronicity and time delay patterns. Physical Review E 66, 4 (Oct 2002), 041904. DOI : https://doi.org/10.1103/ PhysRevE.66.041904

Gongbing Shan and Peter Visentin. 2003. A quantitative three-dimensional analysis of arm kinematics in violin performance. Medical Problems of Performing Artists 18, 1 (2003), 3.

Alessandro Timmi. 2012. Virtual sensei. Retrieved January 17, 2019 from http://www.virtualsensei.it.

Manfred Vieten and Hartmut Riehle. 2005. Movement quality of martial art outside kick. In Proceedings of the XXIII International Symposium on Biomechanics in Sports. 856-860.

Paolo Viviani and Roland Schneider. 1991. A developmental study of the relationship between geometry and kinematics in drawing movements. Journal of Experimental Psychology. Human Perception and Performance 17, 1 (1991), 198-218.

Kerstin Witte, Peter Emmermacher, Nico Langenbeck, and Juergen Perl. 2012. Visualized movement patterns and their analysis to classify similarities demonstrated by the karate kick Mae-geri. Kinesiology: International fournal of Fundamental and Applied Kinesiology 44, 2 (July 2012), 155-165.

Fang Zheng, Guoliang Zhang, and Zhanjiang Song. 2001. Comparison of different implementations of MFCC. fournal of Computer Science and Technology 16, 6 (2001), 582-589. DOI : https://doi.org/10.1007/BF02943243

Received September 2016; revised August 2017; accepted August 2017 